\title{
Non-beta-amyloid/tau cerebrospinal fluid markers inform staging and progression in Alzheimer's disease
}

Umesh Gangishetti ${ }^{1}$, J. Christina Howell ${ }^{1,2}$, Richard J. Perrin ${ }^{3,4}$, Natalia Louneva ${ }^{4}$, Kelly D. Watts ${ }^{1 \wedge}$, Alexander Kollhoff ${ }^{1}$, Murray Grossman ${ }^{7,8,9}$, David A. Wolk ${ }^{6,9}$, Leslie M. Shaw ${ }^{10}$, John C. Morris ${ }^{3,5}$, John Q. Trojanowski ${ }^{6,7,10}$, Anne M. Fagan ${ }^{3,5}$, Steven E. Arnold ${ }^{6,711}$ and William T. Hu ${\text { 1, } 2^{*}}^{\text {(D) }}$

\begin{abstract}
Background: Alzheimer's disease (AD) is a complex neurodegenerative disorder characterized by neuropathologic changes involving beta-amyloid (A $\beta$ ), tau, neuronal loss, and other associated biological events. While levels of cerebrospinal fluid (CSF) $A \beta$ and tau peptides have enhanced the antemortem detection of AD-specific changes, these two markers poorly reflect the severity of cognitive and functional deficits in people with altered $A \beta$ and tau levels. While multiple previous studies identified non- $A \beta$, non-tau proteins as candidate neurodegenerative markers to inform the $A / T / N$ biomarker scheme of $A D$, few have advanced beyond association with clinical $A D$ diagnosis. Here we analyzed nine promising neurodegenerative markers in a three-centered cohort using independent assays to identify candidates most likely to complement $A \beta$ and tau in the $A / T / N$ framework.
\end{abstract}

Methods: CSF samples from 125 subjects recruited at the three centers were exchanged such that each of the nine previously identified biomarkers can be measured at one of the three centers. Subjects were classified according to cognitive status and CSF AD biomarker profiles as having normal cognition and normal CSF $(n=31)$, normal cognition and CSF consistent with AD $(n=13)$, mild cognitive impairment and normal CSF $(n=13)$, mild cognitive impairment with CSF consistent with AD ( $n=23)$, AD dementia ( $n=32$; CSF consistent with AD), and other non-AD dementia ( $n=13$; CSF not consistent with AD).

Results: Three biomarkers were identified to differ among the AD stages, including neurofilament light chain (NfL; $p<0.001$ ), fatty acid binding protein 3 (Fabp3; $p<0.001$ ), and interleukin (IL)-10 ( $p=0.033)$. Increased NfL levels were most strongly associated with the dementia stage of $A D$, but increased Fabp3 levels were more sensitive to milder AD stages and correlated with both CSF tau markers. IL-10 levels did not correlate with tau biomarkers, but were associated with rates of longitudinal cognitive decline in mild cognitive impairment due to $A D(p=0.006)$. Prefreezing centrifugation did not influence measured CSF biomarker levels.

Conclusion: CSF proteins associated with AD clinical stages and progression can complement $A \beta$ and tau markers to inform neurodegeneration. A validated panel inclusive of multiple biomarker features (etiology, stage, progression) can improve AD phenotyping along the A/T/N framework.

Keywords: Biomarkers, Fatty acid binding protein, Interleukin-10, Mild cognitive impairment, Neurofilament light chain

\footnotetext{
* Correspondence: william.hu@emory.edu

'Deceased

'Department of Neurology, Emory University, 615 Michael Street, 505F,

Atlanta, GA 30322, USA

${ }^{2}$ Department of Alzheimer's Disease Research Center, Emory University,

Atlanta, GA, USA

Full list of author information is available at the end of the article
}

(c) The Author(s). 2018 Open Access This article is distributed under the terms of the Creative Commons Attribution 4.0 International License (http://creativecommons.org/licenses/by/4.0/), which permits unrestricted use, distribution, and reproduction in any medium, provided you give appropriate credit to the original author(s) and the source, provide a link to the Creative Commons license, and indicate if changes were made. The Creative Commons Public Domain Dedication waiver (http://creativecommons.org/publicdomain/zero/1.0/) applies to the data made available in this article, unless otherwise stated. 


\section{Background}

The clinicopathologic description of Alzheimer's disease (AD) underwent recent revisions to better characterize, on parallel continuums, the cognitive and neuropathologic features associated with beta-amyloid (A $\beta$ ) deposition, tau hyperphosphorylation, and neurodegeneration [1-5]. This $\mathrm{A} / \mathrm{T} / \mathrm{N}$ framework has the advantage of providing a multidimensional view of $\mathrm{AD}$, although accurate antemortem detection of all three features remains an obstacle in early diagnosis and clinical trial design. AD biomarkers, including cerebrospinal fluid (CSF) [6] or positron emission tomography (PET) $[7,8]$ measures of amyloid and tau proteins, correlate well with postmortem amyloid and tau (A/T) pathology, but their levels have not been shown to accurately track disease progression $[9,10]$ to provide information on neurodegeneration. We and others have previously identified CSF proteins which accompany altered amyloid and tau biomarkers in large discovery cohorts, and these non- $\mathrm{A} \beta$, non-tau (NANT) markers are candidate markers of neurodegeneration [11-17]. However, successful replication of these markers' association with $\mathrm{AD}$ has been challenging. This may be due to many issues, including recruitment bias [18], processing artifacts when assays are performed by commercial vendors [19], and different antibodies, and few of them have been replicated across cohorts and assay platforms to undergo further standardization and application.

CSF is a ready source for simultaneously testing multiple markers reflecting AD core pathology, copathology (ischemia, Lewy bodies), neurodegeneration, common biological alterations (e.g., neuroinflammation), and unique exposures (e.g., environmental toxins) [20]. We previously sought to identify NANT biomarkers through single-center studies $[12,14,19]$, and subsequently determined that some replication failures resulted from biases in recruitment, diagnosis, preanalytical handling, and analytical algorithms [18]. To validate the association between $\mathrm{AD}$ pathology, neurodegeneration, and the top NANT biomarkers, we adapted a round-robin design [21] involving subjects recruited from three Alzheimer's disease centers, and collaboratively measured levels of nine analytes to correlate with $\mathrm{AD}$ biomarkers and clinical AD stages.

\section{Methods}

\section{Standard protocol approvals, registrations, and patient consents}

The protocols were approved by the Institutional Review Boards (IRB) at Emory University (Emory), University of Pennsylvania (Penn), and Washington University (WU). Banked CSF samples were used for this study, and all subjects had previously consented to the long-term storage and subsequent analysis of CSF samples. Frozen CSF samples were exchanged among the centers under six bilateral material transfer agreements.

\section{Subjects and preanalytical processing}

Demographic (age, sex, education), diagnostic (syndrome, global Clinical Dementia Rating (CDR), Mini-Mental State Examination (MMSE)), and APOE allelic information were collected by each center (Table 1). At Emory, CSF was collected via syringe between 08.00 and 12.00 without overnight fasting using 24-G Sprotte needles, placed in polypropylene tubes, and immediately aliquoted without centrifugation, labeled, and frozen at $-80{ }^{\circ} \mathrm{C}$. At Penn, CSF was collected by gravity or syringe without overnight fasting in the morning using 24-G Sprotte needles, placed in polypropylene tubes, transferred locally, aliquoted without centrifugation, labeled, and frozen at $-80^{\circ} \mathrm{C}$. At WU, CSF was collected at 08.00 following overnight fasting via gravity using 22-G Sprotte needles, placed in polypropylene tubes, centrifuged at low speed to pellet any cellular debris, aliquoted, and frozen at $-80{ }^{\circ} \mathrm{C}$. All samples were shipped to the two external sites overnight on dry ice and immediately placed at $-80{ }^{\circ} \mathrm{C}$ until analysis.

\section{Subject grouping}

Each subject was categorized according to clinical diagnosis (normal cognition (NC), mild cognitive impairment/very mild dementia/CDR 0.5 (MCI), AD dementia, and other non-AD dementia (OD)), and those with $\mathrm{NC}$ or MCI were further stratified according to CSF AD biomarkers. In all subjects, CSF levels of $A \beta 42$, total tau (t-Tau), and tau phosphorylated at threonine $181\left(\mathrm{p}-\mathrm{Tau}_{181}\right)$ had been previously measured using INNO-BIA Alzbio3 (Emory [6], Penn [22]) or INNOTEST ${ }^{\circ}$ (WU) [23] following the manufacturer's protocols (Fujirebio US, Malvern, PA). All three centers included subjects with NC without CSF biomarkers consistent with $\mathrm{AD}\left(\mathrm{NC}^{-}\right)$, $\mathrm{MCI}$ with CSF consistent with $\mathrm{AD}\left(\mathrm{MCI}^{+}\right)$, and $\mathrm{AD}$ dementia. In addition, Emory and WU included NC subjects with CSF biomarkers consistent with $\mathrm{AD}\left(\mathrm{NC}^{+}\right)$, and Emory and Penn included MCI subjects with CSF not consistent with $\mathrm{AD}\left(\mathrm{MCI}^{-}\right)$as well as subjects with OD (Table 1). The diagnosis for OD includes behavioral variant frontotemporal dementia $(n=5)$, semantic variant of primary progressive aphasia $(n=1)$, progressive supranuclear palsy $(n=2)$, and dementia with Lewy bodies $(n=5)$.

\section{NANT biomarker assays}

Nine NANT analytes were selected by WTH, AMF, and SEA for validation based on previous biomarker discovery studies, and assay development and performance took place at Emory (interleukin (IL)-7, IL-10, fractalkine, tumor necrosis factor (TNF)- $\alpha$ ), Penn (fatty acid binding protein 3 (Fabp3), insulin-like growth factor binding protein 2 (IGF-BP2), neurofilament light chain (NfL)), and WU (monocyte chemotactic protein 1 (MCP1), chitinase-3-like protein 1 (YKL-40)). At Emory, IL-7, IL-10, fractalkine, and TNF- $\alpha$ levels (Milliplex MAP Human Cytokine Panel, 
Table 1 Demographic features of subjects included in the current study

\begin{tabular}{|c|c|c|c|c|c|c|}
\hline & $\mathrm{NC}^{-}(n=31)$ & $\mathrm{NC}^{+}(n=13)$ & $\mathrm{MCl}^{-}(n=13)$ & $\mathrm{MCl}^{+}(n=23)$ & $\operatorname{AD}$ dementia $(n=32)$ & $\mathrm{OD}(n=13)$ \\
\hline Female, $n(\%)$ & $19(61 \%)$ & $9(69 \%)$ & $7(54 \%)$ & $13(56 \%)$ & $21(66 \%)$ & $5(39 \%)$ \\
\hline Age (years) & $69.1 \pm 6.3$ & $74.6 \pm 6.9$ & $70.1 \pm 5.0$ & $70.6 \pm 6.2$ & $72.8 \pm 7.1$ & $65.4 \pm 4.5$ \\
\hline Caucasian, $n(\%)$ & $28(90 \%)$ & $13(100 \%)$ & $12(92 \%)$ & $23(100 \%)$ & $30(94 \%)$ & $13(100 \%)$ \\
\hline Has at least one APOE $\varepsilon 4$ allele, \% & $26 \%$ & $38 \%$ & $3 / 10(30 \%)$ & $52 \%$ & $72 \%$ & $31 \%$ \\
\hline Education (years) & $15.7 \pm 3.3$ & $15.4 \pm 3.2$ & $15.5 \pm 3.2$ & $15.0 \pm 2.8$ & $14.7 \pm 3.8$ & $15.4 \pm 1.7$ \\
\hline MMSE & $28.9 \pm 1.8$ & $28.5 \pm 1.8$ & $27.4 \pm 2.1$ & $26.4 \pm 2.5$ & $21.7 \pm 4.9$ & $23.2 \pm 6.3$ \\
\hline \multicolumn{7}{|l|}{ Recruiting center, $n$} \\
\hline Emory & 9 & 3 & 7 & 7 & 10 & 8 \\
\hline Penn & 12 & 0 & 6 & 6 & 12 & 5 \\
\hline WU & 10 & 10 & 0 & 10 & 10 & 0 \\
\hline \multicolumn{7}{|l|}{ Emory AD biomarkers (Luminex) } \\
\hline $\mathrm{A} \beta 42(\mathrm{pg} / \mathrm{mL})$ & $301.3 \pm 106.6$ & $189.1 \pm 134.8$ & $306.3 \pm 98.5$ & $184.1 \pm 68.6$ & $171.3 \pm 37.6$ & $232.2 \pm 114.9$ \\
\hline t-Tau (pg/mL) & $48.5 \pm 22.4$ & $77.3 \pm 53.3$ & $63.8 \pm 27.9$ & $150.4 \pm 81.6$ & $148.8 \pm 52.3$ & $90.8 \pm 106.0$ \\
\hline $\mathrm{p}-\mathrm{Tau}_{181}(\mathrm{pg} / \mathrm{mL})$ & $28.0 \pm 10.1$ & $53.4 \pm 26.9$ & $32.5 \pm 6.9$ & $63.3 \pm 19.3$ & $74.7 \pm 16.6$ & $27.6 \pm 15.7$ \\
\hline \multicolumn{7}{|l|}{ Penn AD biomarkers (Luminex) } \\
\hline $\mathrm{A} \beta 42(\mathrm{pg} / \mathrm{mL})$ & $262.6 \pm 72.5$ & N/A & $242.1 \pm 53.1$ & $119.6 \pm 18.0$ & $121.9 \pm 35.0$ & $310.6 \pm 72.9$ \\
\hline t-Tau (pg/mL) & $54.4 \pm 13.1$ & N/A & $76.9 \pm 34.8$ & $96.6 \pm 64.9$ & $117.9 \pm 41.4$ & $66.2 \pm 28.3$ \\
\hline $\mathrm{p}-\mathrm{Tau}_{181}(\mathrm{pg} / \mathrm{mL})$ & $19.3 \pm 14.4$ & N/A & $33.9 \pm 34.2$ & $36.6 \pm 21.1$ & $45.6 \pm 22.6$ & $15.2 \pm 4.5$ \\
\hline \multicolumn{7}{|l|}{ WU AD biomarkers (ELISA) } \\
\hline $\mathrm{A} \beta 42(\mathrm{pg} / \mathrm{mL})$ & $736.9 \pm 152.7$ & $369.2 \pm 87.5$ & N/A & $349.0 \pm 121.8$ & $291.7 \pm 74.4$ & N/A \\
\hline $\mathrm{t}-\mathrm{Tau}(\mathrm{pg} / \mathrm{mL})$ & $282.8 \pm 115.7$ & $359.5 \pm 230.3$ & N/A & $615.2 \pm 177.7$ & $628.2 \pm 363.1$ & N/A \\
\hline $\mathrm{p}-\mathrm{Tau}_{181}(\mathrm{pg} / \mathrm{mL})$ & $54.4 \pm 19.1$ & $74.7 \pm 40.7$ & N/A & $107.4 \pm 52.3$ & $90.7 \pm 52.7$ & N/A \\
\hline
\end{tabular}

Values are shown as mean \pm standard deviation unless otherwise indicated

$A \beta$ beta-amyloid, $A D$ Alzheimer's disease, ELISA enzyme-linked immunosorbent assay, Emory Emory University, $M C I$ mild cognitive impairment, MMSE Mini-Mental State Examination, N/A not available, NC normal cognition, OD other non-AD dementia, Penn University of Pennsylvania, $p$-Tau 181 phosphorylated tau, $t$-Tau total tau, WU Washington University

HCYTOMAG-60 K, EMD Millipore, Billerica, MA) were measured in a Luminex 200 platform following the manufacturer's protocol except that two $100-\mu \mathrm{L}$ aliquots of CSF were used for duplicates. At Penn, plate-based enzyme-linked immunosorbent assays (ELISAs) were performed according to the manufacturer's instructions for human IGFBP-2 (Sigma, St. Louis, MO; cat. no. RAB0233), human FABP3 (EMD Millipore; cat. no. EZFABP3-38 K), and human neurofilament-light RUO (IBL International, Hamburg, Germany; cat. no. UD51001). At WU, MCP1 levels were analyzed in a Luminex 200 platform (Milliplex MAP Human Adipocyte Panel, HADCYMAG-61 K; EMD Millipore, Billerica, MA), and YKL-40 levels were measured using ELISA (MicroVue YKL-40 EIA Kit, Quidel, San Diego, CA) [11]. All operators were blinded to the diagnosis, and final assay results were collected at Emory for analysis.

\section{Effects of centrifugation}

Because CSF samples were centrifuged after collection at WU but not centrifuged at Emory and Penn, we performed prospective experiments at Emory to determine the effect of prefreezing centrifugation. Specifically, after CSF was collected from 16 subjects, CSF samples were immediately divided into two equal portions. One portion was centrifuged at $2000 \mathrm{~g}$ and $4{ }^{\circ} \mathrm{C}$ for $10 \mathrm{~min}$ while the other portion was kept on ice. The supernatant from the centrifuged portion was carefully aliquoted, labeled, and frozen at $-80{ }^{\circ} \mathrm{C}$ until analysis, and the noncentrifuged portion was similarly aliquoted, labeled, and frozen at $80{ }^{\circ} \mathrm{C}$ until analysis. Levels of two analytes whose levels varied according to center (IL-7, IL-10) and one analyte whose level did not vary according to center (NfL) were analyzed in samples with and without centrifugation.

\section{Statistical analysis}

Statistical analysis was performed by IBM-SPSS 24 (Chicago, IL) at Emory. For baseline comparison among the three centers, Chi-squared tests for categorical variables and analysis of variance (ANOVA) for continuous variables were used to determine differences. APOE genotyping was not available for $3 \mathrm{MCI}^{-}$subjects from Emory. Since $\mathrm{MCI}^{-}$ and $\mathrm{OD}$ were included for comparative purposes, these 
missing genotypes did not influence the study's main analysis.

For biomarker levels, ANOVA showed that three analytes (IL-7, IL-10, and MCP-1) differed significantly among the recruiting centers. To standardize data handling and to account for these center-associated differences, a site-specific $Z$ score was created for each analyte using the mean and standard deviation of the combined $\mathrm{NC}^{-}$and AD dementia cohort. After $Z$ transformation, the levels of each analyte were confirmed to be normally distributed by Kolmogorov-Smirnov tests. Student's $t$ tests were then performed to identify analytes whose levels differed between $\mathrm{NC}^{-}$and $\mathrm{AD}$ dementia, with a false discovery rate (FDR) threshold of 0.10 to account for multiple comparisons. Student's $t$ tests were also used to determine whether prefreezing centrifugation affected biomarker levels.

Analysis of covariance (ANCOVA) was used to determine biomarkers that can differentiate among the four theoretical stages of $\mathrm{AD}$ development $\left(\mathrm{NC}^{-}, \mathrm{NC}^{+}, \mathrm{MCI}^{+}, \mathrm{AD}\right.$ dementia), adjusting for age, sex, $A P O E \varepsilon 4$ status, and recruiting center. A threshold of 0.10 for FDR was selected to account for multiple comparisons. Pearson's correlation was then used to analyze the relationships between established CSF AD biomarkers (A $\beta 42$, $t$-Tau, $\left.p-T_{10 u}{ }_{181}\right)$ and the three biomarkers identified through ANCOVA.

Finally, for correlation between baseline IL-10 levels and rates of longitudinal cognitive decline, mixed linear modeling was used to determine whether IL-10 levels were associated with faster rates of cognitive decline. $Z$ scores for executive, memory, language, and visual spatial domains were calculated as previously described. In the mixed linear model, domain-specific $Z$ scores were entered as the dependent variable; gender, race, $\mathrm{t}$-Tau (previously found to influence rates of cognitive decline) [24], IL-10, time, time $\times$ IL-10, age, and education were entered as fixed variables, and time was also entered as a random variable. IL-10 was considered to significantly influence the rates of longitudinal decline if the interaction term time $\times$ IL-10 was associated with domain-specific $Z$ scores at $p<0.01$ to adjust for multiple comparisons.

\section{Results}

The overall cohort included 125 subjects, including 31 $\mathrm{NC}^{-}, 13 \mathrm{NC}^{+}, 12 \mathrm{MCI}^{-}, 24 \mathrm{MCI}^{+}, 32 \mathrm{AD}$ dementia, and 13 OD. Subjects were younger (68.7 vs. 73.4 years, $p=$ 0.003 ) and more educated (15.7 vs. 14.0 years, $p=0.025$ ) at Emory than WU. Neither site differed from Penn. All three sites were otherwise similar for $\operatorname{sex}(p=0.564)$, race $(p=0.418)$, and APOE \&4 status $(p=0.445)$.

\section{NANT biomarkers associated with AD dementia}

Since prior NANT biomarker studies sought biomarkers that distinguished between subjects with $\mathrm{NC}\left(\mathrm{NC}^{-}\right.$with or without $\mathrm{NC}^{+}$) and $\mathrm{AD}$ dementia, we first analyzed whether levels of the nine candidate biomarkers differed between $\mathrm{NC}^{-}$and $\mathrm{AD}$ dementia. This identified three analytes (NfL, Fabp3, and YKL-40) associated with AD dementia after adjusting for FDR of 5\% (Fig. 1). None of the other analytes differed between $\mathrm{NC}^{-}$and $\mathrm{AD}$ dementia (adjusted $p$ value range of 0.252 to 0.977 ). Controlling for age, sex, center of recruitment, and $A P O E \& 4$ status slightly diminished the significance of YKL-40 $(p=0.062)$ but showed similar results for NfL $(p<0.001)$ and Fabp3 $(p<0.001)$. Thus, NfL and Fabp3 best distinguished between the two extreme categories ( $\mathrm{NC}^{-}$and $\mathrm{AD}$ dementia).

\section{NANT biomarkers associated with AD stages}

Since the clinical manifestation of AD neuropathology is hypothesized to progress through the presymptomatic, $\mathrm{MCI}$, and dementia stages, we next examined in this cross-sectional cohort whether levels of the candidate analytes differed among $\mathrm{NC}^{-}, \mathrm{NC}^{+}, \mathrm{MCI}^{+}$, and $\mathrm{AD}$ dementia through ANCOVA adjusting for age, sex, education, and presence of $A P O E$ \&4 allele. This confirmed NfL $(\mathrm{F}(3,94)=9.455, p<0.001)$ and Fabp3 $(\mathrm{F}(3,94)=$ $5.869, p<0.001)$ to be associated with AD stages. Specifically, NfL levels were higher in $\mathrm{AD}$ dementia than $\mathrm{NC}^{-}$, $\mathrm{NC}^{+}$, or $\mathrm{MCI}^{+}$(Fig. 2a), and Fabp3 levels were higher in $\mathrm{AD}$ dementia than $\mathrm{NC}^{-}$and $\mathrm{NC}^{+}$, and higher in $\mathrm{MCI}^{+}$ than $\mathrm{NC}^{-}$(Fig. 2b). Furthermore, IL-10 $(\mathrm{F}(3,94)=3.034$, $p=0.033)$ showed stage-associated differences, with $\mathrm{NC}^{+}$ having lower IL-10 levels than $\mathrm{NC}^{-}$, but $\mathrm{AD}$ dementia having higher IL-10 levels than $\mathrm{NC}^{+}$or $\mathrm{MCI}^{+}$(Fig. 2c). No biomarkers significantly differed in their level between $\mathrm{NC}^{+}$and $\mathrm{MCI}^{+}$(Fig. 2d).

\section{$\mathrm{NfL}$ and Fabp3 levels associated with CSF tau biomarkers}

As emerging $\mathrm{AD}$ therapeutics often target amyloid and tau, levels of established CSF AD biomarkers (A $\beta 42$, $t$-Tau, and $\mathrm{p}-\mathrm{Tau}_{181}$ ) may serve better to inform target engagement than treatment-associated downstream effects. We therefore analyzed if CSF NfL, Fabp3, and IL-10 correlated with the established CSF AD biomarkers (A $\beta 42$, $t-T a u$, and p-Tau $\left.{ }_{181}\right)$ to serve as downstream markers. Analyzing samples from Emory and Penn (where established biomarker assays had been performed on identical Luminex platforms) together, both CSF NfL and Fabp3 levels correlated strongly with CSF t-Tau levels $(p<0.001)$, and CSF Fabp3 $\left(R^{2}=0.348, p<0.001\right)$ levels better correlated with CSF p-Tau ${ }_{181}$ levels than CSF NfL levels $\left(R^{2}=0.069, p=0.035\right.$, not significant after correction for multiple comparisons; Fig. 3). A similar trend was seen in samples from WU (where established biomarker assays were performed by ELISA), with Fabp3 levels correlating with t-Tau $(p<0.001)$ and p-Tau $(p<0.001)$, and NfL levels correlating better with t-Tau $(p<0.001)$ than $\mathrm{p}-\mathrm{Tau}_{181}(p=0.074)$. None of the NANT biomarkers correlated with CSF A $\beta 42$, and diagnosis did not influence the relationship between tau 


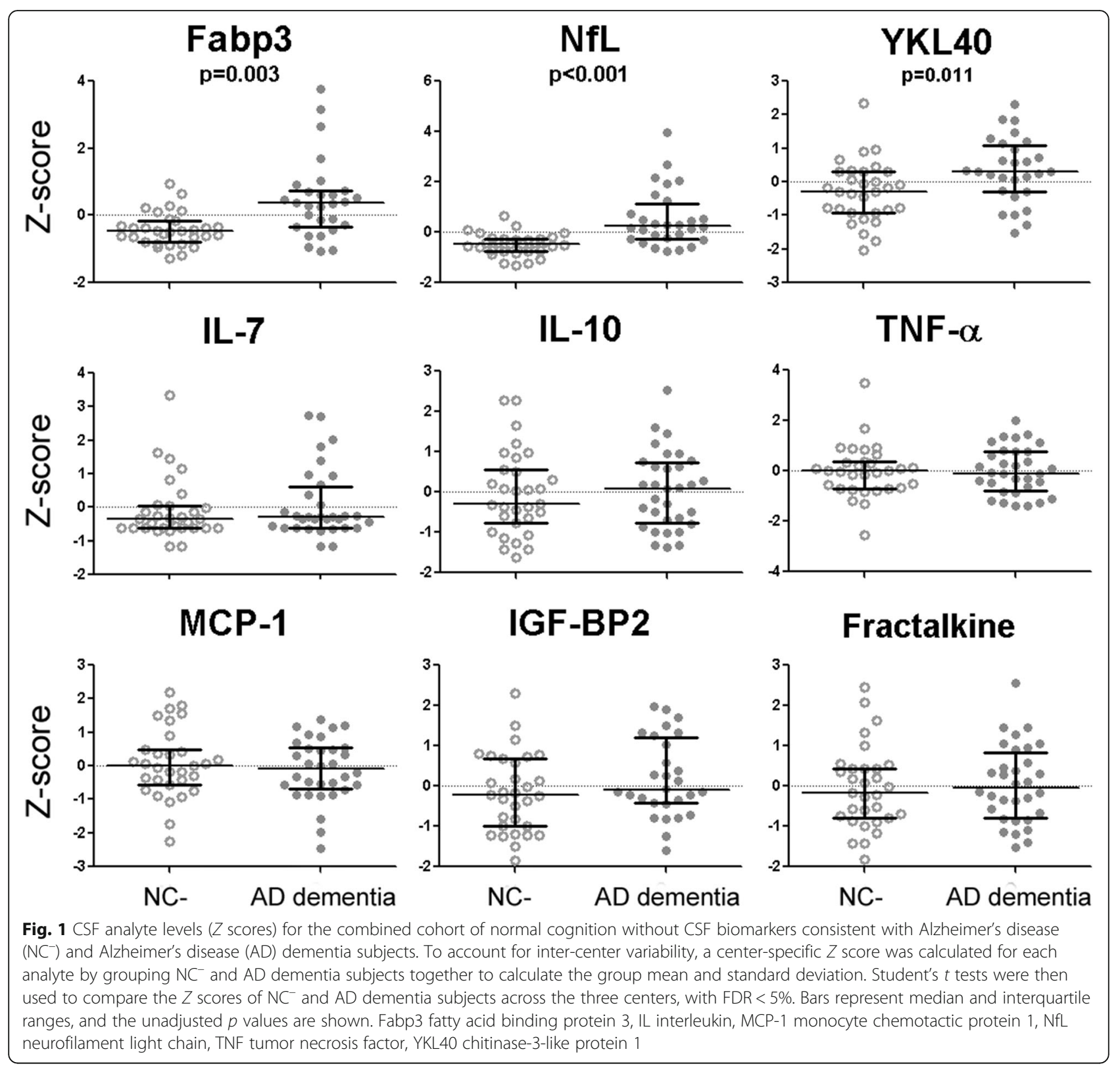

biomarkers and the two novel biomarkers (Fabp3 and NfL).

\section{IL-10 associated with rates of longitudinal cognitive decline in $\mathrm{MCl}^{+}$}

Because CSF IL-10 levels did not correlate with $\mathrm{t}$-Tau or p-Tau ${ }_{181}$, we then analyzed if CSF IL-10 levels correlated with rates of decline in $\mathrm{MCI}^{+}$subjects since clinicians often consider longitudinal decline as an important feature of $\mathrm{MCI}^{+}$. This may introduce bias into the selection of $\mathrm{MCI}^{+}$subjects, especially when IL-10 levels did not differ between $\mathrm{NC}^{-}$and $\mathrm{AD}$. Mixed linear modeling showed that, in a group of $51 \mathrm{MCI}^{+}$subjects longitudinally followed at Emory (median follow-up 36 months, range 18-78 months), lower IL-10 levels were associated with greater rates of decline in memory $Z$ scores $(p=0.006$ for time $\times$ IL-10 levels; Table 2 and Fig. 4a), but not in executive $(p=0.270)$, language $(p=0.246)$, or visual spatial $(p=0.975) Z$ scores. In comparison, higher CSF $\mathrm{t}$-Tau levels were associated with worse memory $Z$ scores, but neither CSF $\mathrm{t}$-Tau nor $\mathrm{p}$-Tau ${ }_{181}$ influenced the rates of memory decline.

\section{NANT biomarker levels not associated with prefreezing CSF centrifugation}

Finally, we sought to determine whether prefreezing CSF centrifugation (performed at WU) represented another bias in measured NANT levels since centrifuged samples 


\section{A NfL $\quad$ B Fabp3}
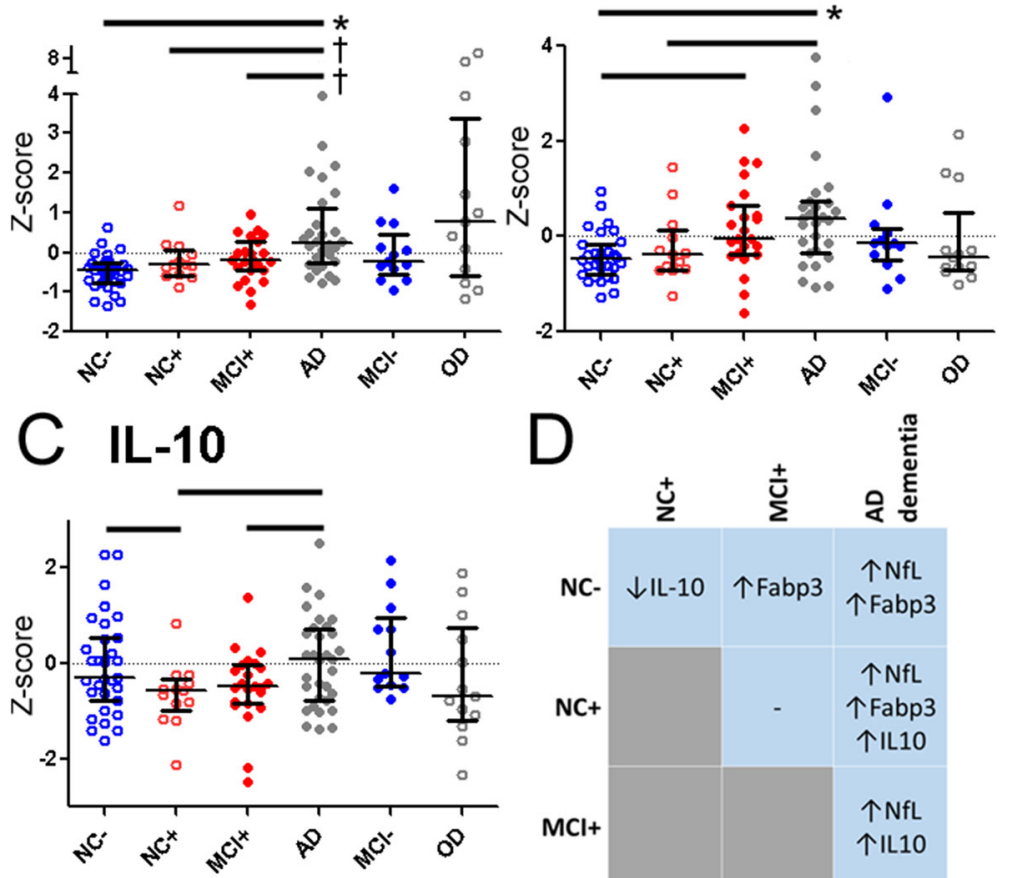

Fig. 2 CSF levels (Z scores) of neurofilament light chain (NfL) (a), fatty acid binding protein 3 (Fabp3) (b), and interleukin (IL)-10 (c) in subjects with normal cognition (NC), mild cognitive impairment (MCI), Alzheimer's disease (AD) dementia, and other non-AD dementia (OD). $\left(^{*} p<0.001\right.$; ${ }^{\dagger} p<0.005 ; p<0.05$ for other comparisons indicated). Differences between different subgroups are summarized in $\mathbf{d}$, with direction of change reflecting the stage with more severe pathology or cognitive impairment

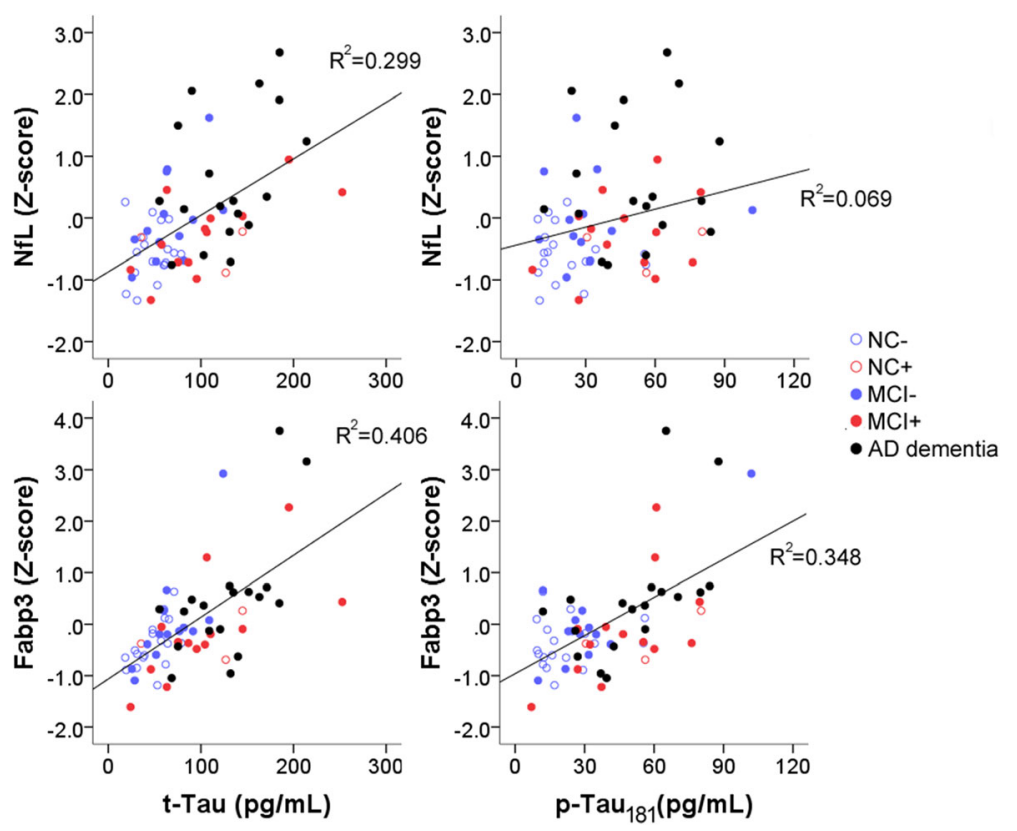

Fig. 3 Correlations between CSF tau-related proteins and neurofilament light chain (NfL) and fatty acid binding protein 3 (Fabp3) levels. Fabp3 levels correlated strongly with total tau (t-Tau) and phosphorylated tau $\left(\mathrm{p}-\mathrm{Tau}_{181}\right)$ levels, while Nfl levels correlated better with t-Tau than $p$-Tau ${ }_{181}$ levels. AD Alzheimer's disease, MCl mild cognitive impairment, NC normal cognition 
Table 2 Mixed linear model analysis of memory $Z$ scores in $\mathrm{MCl}^{+}$subjects longitudinally characterized at Emory $(n=51)$

\begin{tabular}{lll}
\hline & $\begin{array}{l}\text { Coefficient }(95 \% \\
\text { confidence interval) }\end{array}$ & $p$ \\
\hline Age & $0.020(-0.011,0.051)$ & 0.210 \\
Male gender & $0.613(0.148,1.078)$ & 0.011 \\
Minority race & $0.210(-0.647,1.067)$ & 0.625 \\
Education (years) & $0.029(-0.057,0.116)$ & 0.505 \\
t-Tau (pg/mL) & $-0.002(-0.005,-0.001)$ & 0.073 \\
IL-10 level (pg/mL) & $-0.035(-0.139,0.069)$ & 0.510 \\
Time (months) & $-0.043(-0.060,-0.026)$ & $<0.001$ \\
Time $\times$ IL-10 (months $\times \mathrm{pg} / \mathrm{mL})$ & $0.003(0.001,0.005)$ & 0.006 \\
\hline
\end{tabular}

IL interleukin, $\mathrm{MCl}$ mild cognitive impairment, $t$-Tau total tau

represented $77 \%$ of $\mathrm{NC}^{+}$and $43 \%$ of $\mathrm{MCI}^{+}$cases. Centrifuged and noncentrifuged samples prospectively collected from the same individuals at Emory showed similar absolute levels of IL-10 and NfL (Fig. 4b), suggesting that their association with $\mathrm{AD}$ stages was independent of the preanalytical processing differences between the centers. In keeping with this, levels of IL-7 (which showed a large inter-site difference) were also not influenced by centrifugation.

\section{Discussion}

Reproducible NANT biomarkers associated with $\mathrm{AD}$ pathogenesis or progression have the potential for complementing existing cognitive/functional assessments and improving clinical trial designs. Here we used multicentered samples and independent assays to confirm CSF Fabp3 and NfL as stage-dependent biomarkers in AD. The levels of these two markers also correlated with t-Tau (both) and p-Tau ${ }_{181}$ (Fabp3) in the CSF, and can be prospectively tested as surrogate markers of response in future clinical trials targeting tau. Furthermore, we found a complex relationship between CSF IL-10 levels, AD, and cognition, but associated lower CSF IL-10 levels to faster cognitive decline in MCI. Altogether, these findings point to a set of unique biochemical events associated with cumulative and on-going cognitive decline in $\mathrm{AD}$, and add a suite of NANT biomarkers to the A/T/N scheme.

Previous work-including our own-has primarily focused on NANT biomarkers whose levels differed between $\mathrm{NC}^{-}$and $\mathrm{AD}$ dementia. Subjects with normal cognition but abnormal AD biomarkers (CSF or PET) were variably included with or excluded from those whose cognition and AD biomarkers were both normal, and the distinction between $\mathrm{MCI}^{+}$and $\mathrm{AD}$ dementia could be based on the number of impaired neuropsychological domains, functional independence, or consensus. Aside from these study design biases, our current study showed that analyzing only the two extreme groups overlooked at least one biologically meaningful marker,

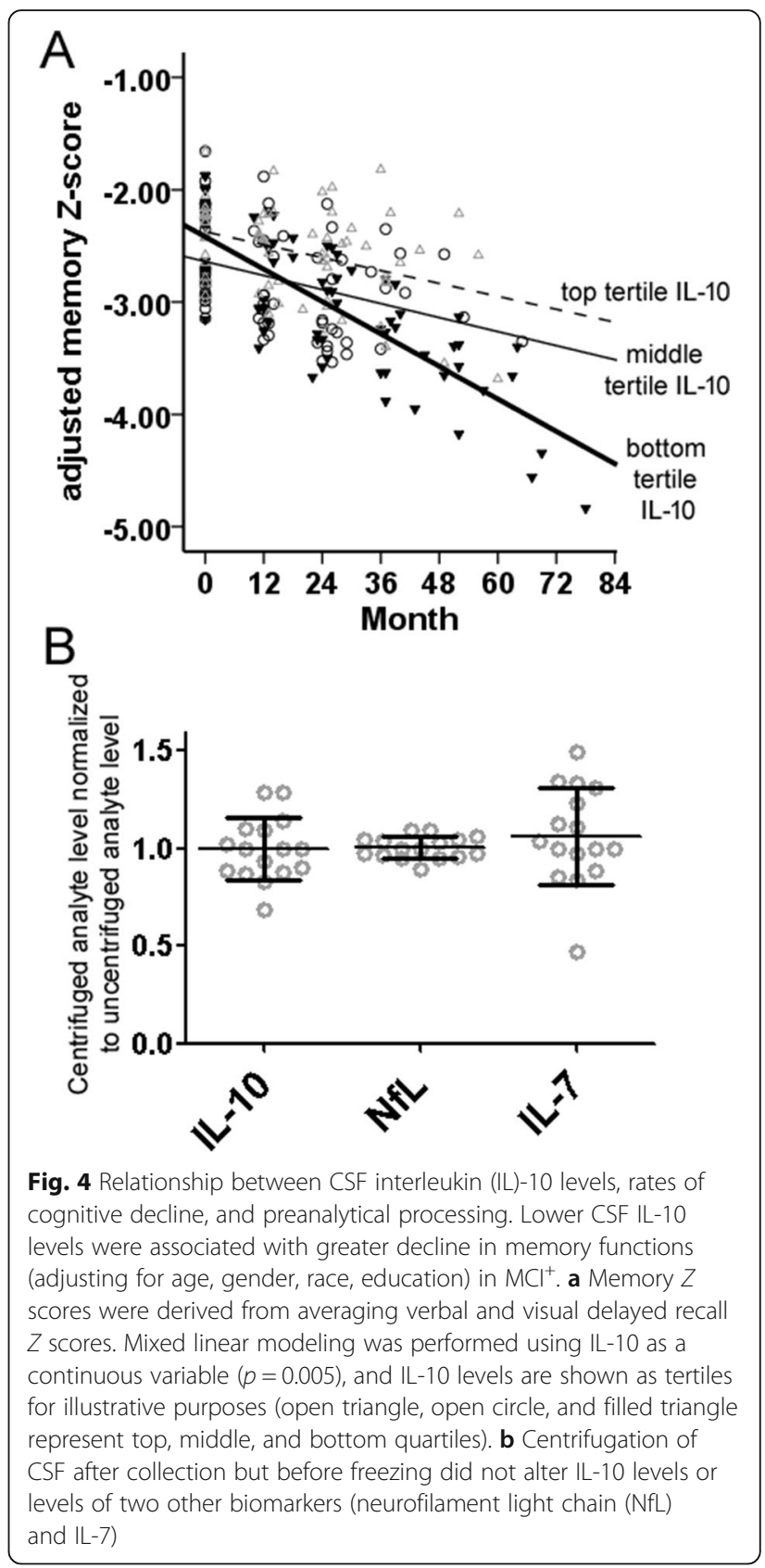

IL-10. At the same time, levels of the most commonly cited candidate staging marker-NfL, a neuronal cytoskeletal protein associated with axonal injury-were most elevated in the dementia stage of $\mathrm{AD}$, but did not sufficiently distinguish between the earlier stages $\left(\mathrm{NC}^{-}, \mathrm{NC}^{+}, \mathrm{MCI}^{+}\right)$ nor correlate strongly with $\mathrm{p}-\mathrm{Tau}_{181}$. The difference in NfL observed here is in line with findings from the Alzheimer's Disease Neuroimaging Initiative (ADNI) and favors NfL more as a marker of staging/progression in neurodegenerative disorders with faster progression (e.g., frontotemporal dementia) than typical AD. Similarly, the difference in YKL-40 levels (a glycoprotein secreted by astrocytes and 
infiltrating macrophages) was consistent with previously reported ranges [11].

Fabp3 levels better distinguished between different AD stages than NfL and YKL-40 [11], and may serve as a good neurodegenerative biomarker since its levels correlated well with CSF t-Tau and p-Tau ${ }_{181}$ levels. Fabp3 is a small soluble protein expressed in neurons, astrocytes, and brain endothelial cells [25-27]. It is involved in the intracellular transport of polyunsaturated fatty acid [28] as well as modulation of acetylcholine and glutamate release [29]. Brains with AD and schizophrenia were found to have reduced Fabp3 levels [30, 31], and serum Fabp3 levels are elevated in multiple dementia and brain injury syndromes [32-34]. Data from the ADNI and other studies have shown that CSF Fabp3 levels do not differ between $\mathrm{NC}^{-}$and $\mathrm{NC}^{+}[35,36]$, but do increase in the symptomatic $\mathrm{AD}$ stages $[36,37]$ and with progressive entorhinal atrophy [38]. Consistent with these prior findings, we also found similar Fabp3 levels in $\mathrm{MCI}^{+}$and AD dementia. Thus, whereas increased NfL levels may reflect sufficient neurodegeneration to result in functional decline [39], Fabp3 may be a more sensitive marker to predementia neurodegeneration.

We found IL-10 levels to differ between clinical AD stages but not between $\mathrm{NC}^{-}$and $\mathrm{AD}$ dementia. This came as counter-intuitive for us, which led to further experiments related to IL-10. CSF IL-10 levels were variably linked with $\mathrm{AD}$ in previous discovery-based studies $[12,14]$. Among potential explanations for these discrepant findings, we eliminated analytical and preanalytical variabilities as confounds in our study since IL-10 levels were all measured at a single site and did not differ in a prospective follow-up study targeting the effects of pre-freezing centrifugation. At the same time, selection bias in banked biospecimens may account for reduced IL-10 levels in $\mathrm{MCI}^{+}$ compared with $\mathrm{AD}$ as lower IL-10 levels were associated with greater rates of memory decline, a feature often considered when MCI samples are selected retrospectively. This is supported by our follow-up study where MCI subjects with the lowest CSF IL-10 levels tended to experience greater memory decline. At the same time, there exist potential biological explanations for lower IL-10 levels in $\mathrm{NC}^{+}$ and $\mathrm{MCI}^{+}$. IL-10 has often been considered an anti-inflammatory cytokine, but it is released by proinflammatory, anti-inflammatory, and regulatory $\mathrm{T}$ helper cells. Its release and effects are thus complex, and IL-10 does not exist or act in isolation. Lower IL-10 levels in $\mathrm{NC}^{+}$may be interpreted as a failure in anti-inflammatory processes associated with onset of pathologic $\mathrm{AD}$, or alternatively balanced anti- and proinflammatory responses in asymptomatic $\mathrm{AD}$ (e.g., we previously showed complement activation to accompany the $\mathrm{MCI}^{+}$to $\mathrm{AD}$ transition [18]). Similarly, higher IL-10 levels in $\mathrm{AD}$ than $\mathrm{NC}^{+}$and $\mathrm{MCI}^{+}$ may represent exaggerated anti-inflammatory responses or appropriate IL-10 response to AD-related neuroinflammation. These challenges call for the simultaneous measurements of cytokines representing different pro- and anti-inflammatory pathways in future studies, as well as immunophenotyping analysis in the CSF. This approach will also better explain why reduced IL-10 levels may predict faster rates of decline in MCI.

Instead of measuring promising $\mathrm{AD}$ biomarkers only at a single site (academic or commercial), we show here that a collaborative model of replication moves the most promising NANT biomarkers towards further development. It enables a greater number of candidate markers to undergo simultaneous validation in subjects recruited from each center in a head-to-head design, identifies analytes with inter-site variabilities, and permits follow-up experiments to empirically determine the effects of different preanalytical procedures. At the same time, our study is limited by the sample size, as yet unidentified factors to account for center-to-center variations, genetic background of populations at the three geographically separate sites, and imperfect matching of some diagnostic categories among centers $\left(\mathrm{NC}^{+}, \mathrm{OD}\right)$. We did not include CSF biomarkers for non-beta-amyloid/tau neurodegenerative processes (e.g., a-synuclein, phosphorylated TDP-43 levels) as they are less mature, and accounting for them may help explain variability across centers and $\mathrm{AD}$ stages. We also did not analyze the NANT biomarker levels in a large group of OD since cases with high confidence pathology (through autopsy confirmation or, less preferably, mutation because of the mutations' potential direct impact on inflammation) are limited in number. Translation of promising markers validated here into the $\mathrm{A} / \mathrm{T} / \mathrm{N}$ biomarker suite will need to prospectively determine the impact of biological, preanalyti$\mathrm{cal}$, and analytical variabilities on the levels and stability of these markers, and the $\mathrm{A} / \mathrm{T} / \mathrm{N}$ scheme itself may need future revision to account for copathology and other contributors.

\section{Conclusion}

In summary, we successfully confirmed three proteins (Fabp3, NfL, and IL-10) as potentially informative biomarkers to complement established $\mathrm{AD}$ biomarkers $\mathrm{A} \beta$ and tau) through a three-centered, North American, non-ADNI study. Importantly, we used assays easily accessible to investigators who can further optimize their development and translation.

\section{Abbreviations}

A $\beta$ : Beta-amyloid; AD: Alzheimer's disease; ADNI: Alzheimer's Disease Neuroimaging Initiative; ANCOVA: Analysis of covariance; ANOVA: Analysis of variance; CDR: Clinical Dementia Rating; CSF: Cerebrospinal fluid; ELISA: Enzyme-linked immunosorbent assay; Emory: Emory University; Fabp3: Fatty acid binding protein 3; FDR: False discovery rate; IGFBP2: Insulin-like growth factor binding protein 2; IL: Interleukin; MCl: Mild cognitive impairment; MCP1: Monocyte chemotactic protein 1; MMSE: MiniMental State Examination; NANT: Non-beta-amyloid, non-tau; NC: Normal 
cognition; NfL: Neurofilament light chain; OD: Other non-Alzheimer's disease dementia; Penn: University of Pennsylvania; PET: Positron emission tomography; $\mathrm{p}-\mathrm{Tau}_{181}$ : Tau phosphorylated at threonine 181; TNF: Tumor necrosis factor; t-Tau: Total tau; WU: Washington University; YKL40: Chitinase-3-like protein 1 (Chi3-I1)

\section{Acknowledgements}

The authors wish to acknowledge Allan I. Levey, MD, PhD, James J. Lah, MD, PhD, David M. Holtzman, MD, Jason H. Karlawish, MD, and Vivianna Van Deerlin, MD, PhD, and the National Alzheimer's Coordinating Center for funding, collecting data, and general support.

\section{Funding}

This work was supported by the National Institutes of Health (AG43885, AG42856, AG25688, AG10124, AG17586, AG05681, AG26276, AG03991, AG16976).

\section{Availability of data and materials}

The datasets used and/or analyzed during the current study available from the corresponding author on reasonable request.

\section{Authors' contributions}

UG, RJP, LMS, JCM, JQT, AMF, SEA, and WTH were responsible for conception and design of the study; UG, JCH, RJP, NL, KDW, AK, MG, DAW, LMS, JCM, JQT, AMF, SEA, and WTH were responsible for acquisition, analysis, and interpretation of data; UG, JCH, JQT, AMF, SEA, and WTH were responsible for drafting the manuscript and revising it critically for important intellectual content. All authors read and approved the final manuscript.

\section{Ethics approval and consent to participate}

This study was approved by Institutional Review Boards at Emory University, University of Pennsylvania, and Washington University. All participants previously consented to CSF storage and analysis of stored CSF samples.

\section{Consent for publication}

Not applicable.

\section{Competing interests}

LMS has received personal compensation for activities with Roche Diagnostics which produces CSF amyloid and tau assays. AMF is on the Scientific Advisory Boards for Roche Diagnostics which produces CSF amyloid and tau assays. WTH consults for ViveBio, LLC., which manufactures lumbar puncture trays; has a patent (assignee: Emory University) on the use of CSF p/t-Tau ratio in the evaluation of FTLD; has received research support from Fujirebio USA and Avid Pharmaceuticals; has received travel support from Hoffman La Roche and Abbvie. The remaining authors declare that they have no competing interests,

\section{Publisher's Note}

Springer Nature remains neutral with regard to jurisdictional claims in published maps and institutional affiliations.

\section{Author details}

${ }^{1}$ Department of Neurology, Emory University, 615 Michael Street, 505F, Atlanta, GA 30322, USA. ²Department of Alzheimer's Disease Research Center, Emory University, Atlanta, GA, USA. ${ }^{3}$ Knight Alzheimer's Disease Research Center, Washington University, St. Louis, MO, USA. ${ }^{4}$ Department of Pathology, Washington University, St. Louis, MO, USA. ${ }^{5}$ Department of Neurology, Washington University, St. Louis, MO, USA. ${ }^{6}$ Penn Memory Center, University of Pennsylvania, Philadelphia, PA, USA. ${ }^{7}$ Center for Neurodegenerative Disease Research, University of Pennsylvania, Philadelphia, PA, USA. ${ }^{8}$ Penn FTD Center, University of Pennsylvania, Philadelphia, PA, USA. 'Department of Neurology, University of Pennsylvania, Philadelphia, PA, USA. ${ }^{10}$ Department of Pathology and Laboratory Medicine, University of Pennsylvania, Philadelphia, PA, USA. ${ }^{11}$ Present Address: Massachusetts General Hospital, Boston, MA, USA.
Received: 14 May 2018 Accepted: 3 September 2018 Published online: 25 September 2018

\section{References}

1. Sperling RA, Aisen PS, Beckett LA, Bennett DA, Craft S, Fagan AM, Iwatsubo T, Jack CR Jr, Kaye J, Montine TJ, et al. Toward defining the preclinical stages of Alzheimer's disease: recommendations from the National Institute on Aging-Alzheimer's Association workgroups on diagnostic guidelines for Alzheimer's disease. Alzheimers Dement. 2011;7:280-92.

2. Albert MS, DeKosky ST, Dickson D, Dubois B, Feldman HH, Fox NC, Gamst A, Holtzman DM, Jagust WJ, Petersen RC, et al. The diagnosis of mild cognitive impairment due to Alzheimer's disease: recommendations from the National Institute on Aging-Alzheimer's Association workgroups on diagnostic guidelines for Alzheimer's disease. Alzheimers Dement. 2011;7:270-9.

3. McKhann GM, Albert MS, Grossman M, Miller B, Dickson D, Trojanowski JQ. Clinical and pathological diagnosis of frontotemporal dementia: report of the Work Group on Frontotemporal Dementia and Pick's Disease. Arch Neurol. 2001;58:1803-9.

4. Hyman BT, Phelps CH, Beach TG, Bigio EH, Cairns NJ, Carrillo MC, Dickson DW, Duyckaerts C, Frosch MP, Masliah E, et al. National Institute on AgingAlzheimer's Association guidelines for the neuropathologic assessment of Alzheimer's disease. Alzheimers Dement. 2012;8:1-13.

5. Jack CR Jr, Bennett DA, Blennow K, Carrillo MC, Dunn B, Haeberlein SB, Holtzman DM, Jagust W, Jessen F, Karlawish J, et al. NIA-AA research framework: toward a biological definition of Alzheimer's disease. Alzheimers Dement. 2018;14:535-62.

6. Hu WT, Watts KD, Shaw LM, Howell JC, Trojanowski JQ, Basra S, Glass JD, Lah JJ, Levey Al. CSF beta-amyloid 1-42-what are we measuring in Alzheimer's disease? Ann Clin Transl Neurol. 2015;2:131-9.

7. Clark CM, Schneider JA, Bedell BJ, Beach TG, Bilker WB, Mintun MA, Pontecorvo MJ, Hefti F, Carpenter AP, Flitter ML, et al. Use of florbetapir-PET for imaging beta-amyloid pathology. Jama. 2011;305:275-83.

8. Marquie M, Siao Tick Chong M, Anton-Fernandez A, Verwer EE, SaezCalveras N, Meltzer AC, Ramanan P, Amaral AC, Gonzalez J, Normandin MD, et al. [F-18]-AV-1451 binding correlates with postmortem neurofibrillary tangle Braak Staging. Acta Neuropathol. 2017;134(4):619-28.

9. Bertens D, Knol DL, Scheltens P, Visser PJ, Alzheimer's Disease Neuroimaging Initiative. Temporal evolution of biomarkers and cognitive markers in the asymptomatic, $\mathrm{MCl}$, and dementia stage of Alzheimer's disease. Alzheimers Dement. 2015;11:511-22.

10. Shokouhi S, McKay JW, Baker SL, Kang H, Brill AB, Gwirtsman HE, Riddle WR, Claassen DO, Rogers BP, Alzheimer's Disease Neuroimaging Initiative. Reference tissue normalization in longitudinal (18)F-florbetapir positron emission tomography of late mild cognitive impairment. Alzheimers Res Ther. 2016;8:2.

11. Craig-Schapiro R, Perrin RJ, Roe CM, Xiong C, Carter D, Cairns NJ, Mintun MA, Peskind ER, Li G, Galasko DR, et al. YKL-40: a novel prognostic fluid biomarker for preclinical Alzheimer's disease. Biol Psychiatry. 2010;68:903-12.

12. Hu WT, Chen-Plotkin A, Arnold SE, Grossman M, Clark CM, Shaw LM, Pickering E, Kuhn M, Chen Y, McCluskey L, et al. Novel CSF biomarkers for Alzheimer's disease and mild cognitive impairment. Acta Neuropathol. 2010;119:669-78.

13. Hu WT, Holtzman DM, Fagan AM, Shaw LM, Perrin R, Arnold SE, Grossman M, Xiong C, Craig-Schapiro R, Clark CM, et al. Plasma multianalyte profiling in mild cognitive impairment and Alzheimer disease. Neurology. 2012;79:897-905.

14. Craig-Schapiro R, Kuhn M, Xiong C, Pickering EH, Liu J, Misko TP, Perrin RJ, Bales KR, Soares H, Fagan AM, Holtzman DM. Multiplexed immunoassay panel identifies novel CSF biomarkers for Alzheimer's disease diagnosis and prognosis. PLoS One. 2011;6:e18850.

15. Abdi F, Quinn JF, Jankovic J, McIntosh M, Leverenz JB, Peskind E, Nixon R, Nutt J, Chung K, Zabetian C, et al. Detection of biomarkers with a multiplex quantitative proteomic platform in cerebrospinal fluid of patients with neurodegenerative disorders. J Alzheimers Dis. 2006;9:293-348.

16. Castano EM, Roher AE, Esh CL, Kokjohn TA, Beach T. Comparative proteomics of cerebrospinal fluid in neuropathologically-confirmed Alzheimer's disease and non-demented elderly subjects. Neurol Res. 2006;28:155-63.

17. Hendrickson RC, Lee AY, Song Q, Liaw A, Wiener M, Paweletz CP, Seeburger $J$, Li J, Meng F, Deyanova EG, et al. High resolution discovery proteomics reveals candidate disease progression markers of Alzheimer's disease in human cerebrospinal fluid. PLoS One. 2015;10:e0135365.

18. Hu WT, Watts KD, Tailor P, Nguyen TP, Howell JC, Lee RC, Seyfried NT, Gearing M, Hales CM, Levey Al, et al. CSF complement 3 and factor $\mathrm{H}$ are staging biomarkers in Alzheimer's disease. Acta Neuropathol Commun. 2016;4:14. 
19. Hu WT, Watts K, Grossman M, Glass J, Lah JJ, Hales C, Shelnutt M, Van Deerlin V, Trojanowski JQ, Levey Al. Reduced CSF p-Tau181 to Tau ratio is a biomarker for FTLD-TDP. Neurology. 2013;81:1945-52.

20. Richardson JR, Roy A, Shalat SL, von Stein RT, Hossain MM, Buckley B, Gearing M, Levey Al, German DC. Elevated serum pesticide levels and risk for Alzheimer disease. JAMA Neurol. 2014;71:284-90.

21. Pannee J, Gobom J, Shaw LM, Korecka M, Chambers EE, Lame M, Jenkins R, Mylott W, Carrillo MC, Zegers I, et al. Round robin test on quantification of amyloid-beta 1-42 in cerebrospinal fluid by mass spectrometry. Alzheimers Dement. 2016;12:55-9.

22. Shaw LM, Vanderstichele H, Knapik-Czajka M, Clark CM, Aisen PS, Petersen RC, Blennow K, Soares H, Simon A, Lewczuk P, et al. Cerebrospinal fluid biomarker signature in Alzheimer's disease neuroimaging initiative subjects. Ann Neurol. 2009;65:403-13.

23. Fagan AM, Roe CM, Xiong C, Mintun MA, Morris JC, Holtzman DM. Cerebrospinal fluid tau/beta-amyloid(42) ratio as a prediction of cognitive decline in nondemented older adults. Arch Neurol. 2007;64:343-9.

24. Ben Bouallegue F, Mariano-Goulart D, Payoux P, Alzheimer's Disease Neuroimaging Initiative. Comparison of CSF markers and semi-quantitative amyloid PET in Alzheimer's disease diagnosis and in cognitive impairment prognosis using the ADNI-2 database. Alzheimers Res Ther. 2017;9:32.

25. Veerkamp JH, Paulussen RJ, Peeters RA, Maatman RG, van Moerkerk HT, van Kuppevelt TH. Detection, tissue distribution and (sub)cellular localization of fatty acid-binding protein types. Mol Cell Biochem. 1990;98:11-8.

26. Owada Y, Yoshimoto T, Kondo H. Spatio-temporally differential expression of genes for three members of fatty acid binding proteins in developing and mature rat brains. J Chem Neuroanat. 1996;12:113-22.

27. Teunissen CE, Veerhuis R, De Vente J, Verhey FR, Vreeling F, van Boxtel MP, Glatz $J$, Pelsers MA. Brain-specific fatty acid-binding protein is elevated in serum of patients with dementia-related diseases. Eur J Neurol. 2011;18:865-71.

28. Offner GD, Brecher P, Sawlivich WB, Costello CE, Troxler RF. Characterization and amino acid sequence of a fatty acid-binding protein from human heart. Biochem J. 1988;252:191-8.

29. Shioda N, Yamamoto Y, Watanabe M, Binas B, Owada Y, Fukunaga K. Hearttype fatty acid binding protein regulates dopamine D2 receptor function in mouse brain. J Neurosci. 2010;30:3146-55.

30. Cheon MS, Kim SH, Fountoulakis M, Lubec G. Heart type fatty acid binding protein (H-FABP) is decreased in brains of patients with Down syndrome and Alzheimer's disease. J Neural Transm Suppl. 2003;67:225-34.

31. Hamazaki K, Maekawa M, Toyota T, Iwayama Y, Dean B, Hamazaki T, Yoshikawa T. Fatty acid composition and fatty acid binding protein expression in the postmortem frontal cortex of patients with schizophrenia: a case-control study. Schizophr Res. 2016;171:225-32.

32. Mollenhauer B, Steinacker P, Bahn E, Bibl M, Brechlin P, Schlossmacher MG Locascio JJ, Wiltfang J, Kretzschmar HA, Poser S, et al. Serum heart-type fatty acid-binding protein and cerebrospinal fluid tau: marker candidates for dementia with Lewy bodies. Neurodegener Dis. 2007;4:366-75.

33. O'Bryant SE, Xiao G, Edwards M, Devous M, Gupta VB, Martins R, Zhang F, Barber R, Texas Alzheimer's R, Care C. Biomarkers of Alzheimer's disease among Mexican Americans. J Alzheimers Dis. 2013;34:841-9.

34. Park SY, Kim MH, Kim OJ, Ahn HJ, Song JY, Jeong JY, Oh SH. Plasma hearttype fatty acid binding protein level in acute ischemic stroke: comparative analysis with plasma S100B level for diagnosis of stroke and prediction of long-term clinical outcome. Clin Neurol Neurosurg. 2013;115:405-10.

35. Hoglund K, Kern S, Zettergren A, Borjesson-Hansson A, Zetterberg H, Skoog I, Blennow K. Preclinical amyloid pathology biomarker positivity: effects on tau pathology and neurodegeneration. Transl Psychiatry. 2017;7:e995.

36. Harari O, Cruchaga C, Kauwe JS, Ainscough BJ, Bales K, Pickering EH, Bertelsen S, Fagan AM, Holtzman DM, Morris JC, et al. Phosphorylated tauAbeta42 ratio as a continuous trait for biomarker discovery for early-stage Alzheimer's disease in multiplex immunoassay panels of cerebrospinal fluid. Biol Psychiatry. 2014;75:723-31.

37. Chiasserini D, Parnetti L, Andreasson U, Zetterberg H, Giannandrea D, Calabresi P, Blennow K. CSF levels of heart fatty acid binding protein are altered during early phases of Alzheimer's disease. J Alzheimers Dis. 2010;22:1281-8.

38. Desikan RS, Thompson WK, Holland D, Hess CP, Brewer JB, Zetterberg H, Blennow $K$, Andreassen OA, McEvoy LK, Hyman BT, et al. Heart fatty acid binding protein and Abeta-associated Alzheimer's neurodegeneration. Mol Neurodegener. 2013;8:39.

39. Merluzzi AP, Carlsson CM, Johnson SC, Schindler SE, Asthana S, Blennow K, Zetterberg $\mathrm{H}$, Bendlin BB. Neurodegeneration, synaptic dysfunction, and gliosis are phenotypic of Alzheimer dementia. Neurology. 2018.

Ready to submit your research? Choose BMC and benefit from:

- fast, convenient online submission

- thorough peer review by experienced researchers in your field

- rapid publication on acceptance

- support for research data, including large and complex data types

- gold Open Access which fosters wider collaboration and increased citations

- maximum visibility for your research: over $100 \mathrm{M}$ website views per year

At BMC, research is always in progress.

Learn more biomedcentral.com/submissions 